\title{
Anti-competition of laser modes in semiconductor lasers
}

\author{
Chi-Chia Huang ${ }^{\mathrm{a}}$, Ching-Fuh Lin ${ }^{\mathrm{a}, \mathrm{b}}$, Yu-Chen $\mathrm{Yu}^{\mathrm{c}}$ and Yi-Xiong Lin ${ }^{\mathrm{c}}$ \\ ${ }^{a}$ Graduate Inst. of Electron-Optical Engr., National Taiwan Univ., Taiwan \\ ${ }^{b}$ Dept. of Electrical Engr.and Graduate Inst. of Electronics Engr., National Taiwan Univ., Taiwan \\ ${ }^{\mathrm{c}}$ Industrial Technology of Research Institute, Chutung, Hsinchu, Taiwan
}

\begin{abstract}
Anti-competition of laser modes is observed in dual-wavelength semiconductor lasers with single gain medium. Under anti-competition, the increase of intensity of one lasing mode could enhance the intensity of another mode, which is opposite to the usual mode competition. In our experiment, anti-competition can be observed for wavelength separation larger than $111 \mathrm{~nm}$, and gradually disappears for wavelength separation less than $100 \mathrm{~nm}$. Besides, anti-competition can also be influenced by the intensity and the wavelength position of both modes. A simple theoretical analysis shows that anti-competition is due to the physics similar to optical pumping.
\end{abstract}

Keywords: mode competition, anti-competition, two-mode semiconductor laser, nonidentical multiple quantum wells, broadband semiconductor optical amplifier.

\section{INTRODUCTION}

Mode competition is a well-known phenomenon in lasers. Soon after the invention of lasers, competition of laser modes has been observed and analyzed [1]. In a multi-mode laser system, different modes will in general have different gains, losses and saturation parameters, and will compete for the available population inversion in the laser. Since the total gain of a laser system is constant under fixed external pumping level, oscillation in one mode will generally reduce the gain available for another mode, and in some situations may suppress the other mode entirely [2]. Under strong coupling condition, competition can lead to bistability of the two lasing modes [3], which has been analyzed through perturbation stability analysis [2]. Competition can also lead to tristability $[4,5]$ and even chaotic behaviors $[6,7]$. Those phenomena are found useful for optical switching, optical logic, data encryption [8,9], and so on. Competition dynamics is also an important phenomenon in injection locking [10], mode locking [11-13], and cross gain modulation in optical amplifiers [14]. For many years, the appearance of competition has been taken for granted among laser modes, and is thought to be inevitable. However, we have discovered a behavior opposite to competition between lasing modes. That is, the increase of the oscillation intensity in one lasing mode was found to enhance the intensity of another mode. This behavior is what we called anti-competition of laser modes.

In this work, we will demonstrate that anti-competition can be observed at wavelength separation larger than 111 $\mathrm{nm}$. Besides, the larger the wavelength separation is, the more apparent anti-competition can be observed. Some other influential factors of anti-competition will also be demonstrated, including the intensity and the wavelength position of the two oscillating modes. A simple theoretical analysis will be given, which shows that anti-competition is due to the physics similar to optical pumping.

\section{EXPERIMENT}

\subsection{Broadband gain medium design}

Since anti-competition can be observed only when the WS is larger than $111 \mathrm{~nm}$, a broadband laser gain medium is necessary. Quantum-well (QW) engineering is a convenient, widely used approach to broaden the bandwidth of semiconductor superluminescent diodes (SLDs). This scheme includes using a single QW with simultaneous transitions of $n=1$ and $n=2$ states [15,16], and using nonidentical QWs [17-20]. Because the simultaneous transitions of $n=1$ and $\mathrm{n}=2$ energy states in identical QWs rely strongly on the device lengths $[15,16]$, nonidentical multiple quantum wells (MQWs) were recently been widely used for broadband purposes. 


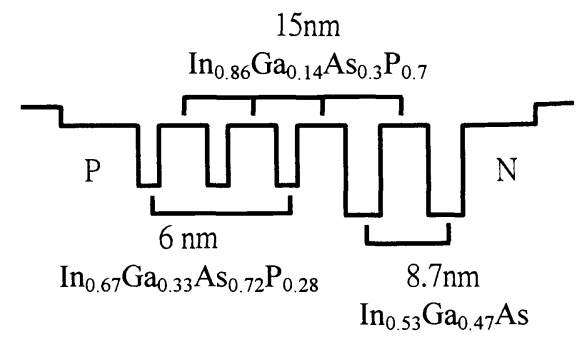

Fig. 1. Designed QW structure.

However, theoretically analysis has predicted that carrier distribution among the MQWs is nonuniform [17, 18]. Experimental evidence of this phenomenon was also indirectly obtained from the characteristics of laser diodes [19, 20]. Nonuniform carrier distribution means that each QW of the MQW structure accumulates a different number of carriers, so their corresponding emission intensities are not equal. Thus, the overlap of the individual spectrum from each type of QW, weighed by its corresponding emission intensity, does not directly result in a broadband spectrum. Investigation on the influential factors of the nonuniform carrier distribution in order to achieve broadband characteristics is thus important [19-21]. With proper design of the nonidentical QW structure, extremely broad bandwidth of the gain medium of the semiconductor lasers/amplifires is possible [22].

The designed QW structure is shown in Fig.1. A separate confinement heterostructure $(\mathrm{SCH})$ is formed in connection with the wells. The $\mathrm{SCH}$ layer has a thickness of $120 \mathrm{~nm}$. The wells are separated by $15-\mathrm{nm}$-wide $\mathrm{In}_{0.86} \mathrm{Ga}_{0.14} \mathrm{As}_{0.3} \mathrm{P}_{0.7}$ barriers. Three 6.0-nm $\operatorname{In}_{0.67} \mathrm{Ga}_{0.33} \mathrm{As}_{0.72} \mathrm{P}_{0.28}$ QWs are placed near the $\mathrm{n}$-cladding layer, and two 8.7$\mathrm{nm} \mathrm{In} \mathrm{I}_{0.53} \mathrm{Ga}_{0.47} \mathrm{As} \mathrm{QWs}$ are placed near the p-cladding layer. The emission energies of the 6.0-nm $\operatorname{In}_{0.67} \mathrm{Ga}_{0.33} \mathrm{As}_{0.72} \mathrm{P}_{0.28}$ $\mathrm{QW}$ and 8.7-nm $\mathrm{In}_{0.53} \mathrm{Ga}_{0.47} \mathrm{As} \mathrm{QW}$ were calculated using the Luttinger-Kohn method [23]. Table I shows the emission wavelengths corresponding to the calculated energy levels. $\operatorname{In}_{0.67} \mathrm{Ga}_{0.33} \mathrm{As}_{0.72} \mathrm{P}_{0.28} \mathrm{QWs}$ and $\mathrm{In}_{0.53} \mathrm{Ga}_{0.47} \mathrm{As}$ QWs have three and two quantized energy levels, respectively.

For this QW structure, emission at low injection current occurs at the wavelength corresponding to the $\mathrm{n}=1$ transition in the 8.7-nm $\operatorname{In}_{0.53} \mathrm{Ga}_{0.47} \mathrm{As}$ QWs, which are close to the n-cladding layer. When the injection current increases, the emission spectrum is broadened owing to the simultaneous transitions of $n=1$ and $n=2$ states. The emission contribution from the $6.0-\mathrm{nm} \mathrm{In}_{0.67} \mathrm{Ga}_{0.33} \mathrm{As}_{0.72} \mathrm{P}_{0.28}$ QWs is obvious only when the injection current is very large. In our experiment, we use laser diodes (LDs) with straight waveguide. This is because, compared with the tilt or bent waveguide superluminescent diodes (SLDs), the straight-waveguide LD has lower loss and lower threshold current. This can facilitate the tuning of the modes above $1500 \mathrm{~nm}$ because those modes are very loss-sensitive. The length of the device was about $300 \mu \mathrm{m}$. No facet coatings were applied to the device. The measured emission spectrum of the LD is shown in Fig. 2. The bandwidth of the emission spectrum is limited by the Fabry-Perot resonance of the straight waveguide LD. The operation temperature and current are $22.7^{\circ} \mathrm{C}$ and $146 \mathrm{~mA}$, respectively. The operation current is only $3 \mathrm{~mA}$ larger than the threshold current of the Fabry-Perot mode, so the oscillation of the Fabry-Perot mode is still negligible.

TABLE I. Calculated Transition Wavelengths Corresponding to the Bounded

\begin{tabular}{c|c|c}
\multicolumn{3}{c}{ Energy States of the Nonidentical MQWs of the Designed SLD } \\
\hline $\mathrm{n}$ & $\begin{array}{c}8.7-\mathrm{nm} \mathrm{In}_{0.53} \mathrm{Ga}_{0.47} \mathrm{As} \\
\text { Double QW }(\mu \mathrm{m})\end{array}$ & $\begin{array}{c}\text { 6.0-nm } \operatorname{In}_{0.67} \mathrm{Ga}_{0.33} \mathrm{As}_{0.72} \mathrm{P}_{0.28} \\
\text { Triple QW }(\mu \mathrm{m})\end{array}$ \\
\hline 1 & 1.54 & 1.3 \\
2 & 1.46 & 1.24 \\
3 & 1.18 & Unbounded \\
\hline
\end{tabular}




\subsection{Experimental setup and steps}

Our experimental setup is shown in Fig. 3. The external cavity is of reflected-type grating telescope configuration. Two collimators with $\mathrm{f}=4.5 \mathrm{~mm}$ and NA $=0.55$ are used to collimate the light beams emitted from two facets of the LD. The coupling efficiency of the collimators is about $70 \%$. The grating is 600 lines $/ \mathrm{mm}$ and is Au-coated. Its efficiency is about $80 \%$. A lens with $\mathrm{f}=10 \mathrm{~cm}$ is placed at $10 \mathrm{~cm}$ from both the grating and the mirror $\mathrm{M} 2$. The insertion of the mirror M1 and two physically separated mirrors for the mirror M2 are for the purpose of broadband tuning range. A double-slit is used for selecting the short-wavelength mode (SWM) and the long-wavelength mode (LWM), and a ND filter is put in front of each slit to control the light power of each mode at the same time.

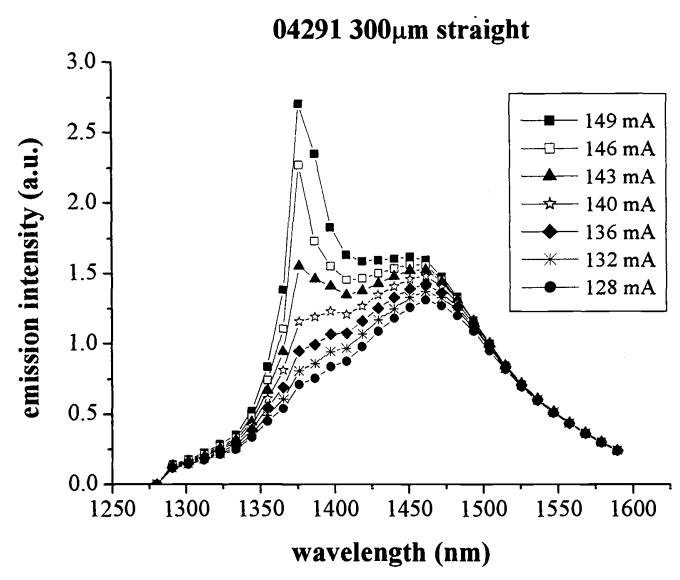

Fig. 2. Measured emission spectrum of the SLD.

Lock-in amp.

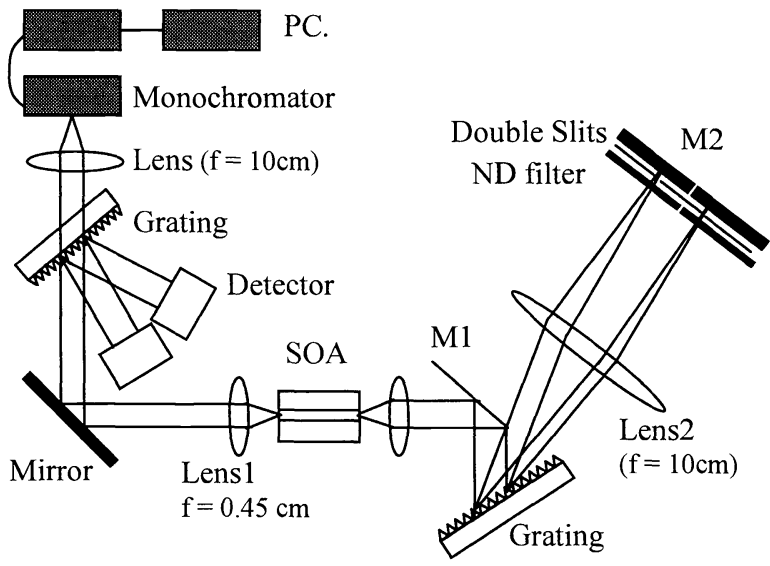

Fig. 3. Experimental setup.

By varying the loss of the ND filter in front of the slit, we can change the intensity of the controlled mode. When the intensity of either mode is changed, the intensity of the other mode also varies due to the competition or anticompetition. Plotting out the variation of the power of those modes, we can obtain several curves on the power plane, where the two axes represent the power of the SWM and the LWM, respectively. Moreover, if the vertical axis represents the power of the LWM, then the external loss introduced to the LWM must be kept constant throughout the measurement, and vice versa. The experiment for obtaining the curve with the vertical axis representing the power of the LWM goes through as follows. First, we choose the double-silt of certain mode spacing. The grating at the output 
diffracts the two oscillation wavelengths chosen by the double-slit, and their light power is measured through two detectors and an oscilloscope. Next, we tune the left ND filter (the one corresponding to the SWM) to a certain value of loss, and again measure the light power of both modes. Because the holding stage of the optical components in the external cavity is not very stable, the ND filter of the LWM is tuned for keeping the external loss a constant if necessary. This step is repeated until we have tuned the ND filter to its maximum loss, and the light power of the SW mode is about to vanish. After that, we have obtained a curve with certain external loss, or say, certain initial power of the LWM. Then, we tune the ND filter of the LWM to change the external loss, and follow the steps mentioned above to obtain another curve with this new external loss and initial power of the LWM. Finally, we remove the grating and the detectors and use the monochromator to measure the spectrum. For obtaining the curves with vertical axis represents the power of the SWM, the experimental steps are similar to that described above.

\subsection{Experimental results and discussion}

Our experimental result for wavelength separation $18 \mathrm{~nm}$ is shown in Fig. 4 (a). We see that, under this wavelength separation, the curves are of negative gradient. That is, the light power of the LWM decreases with the increasing of the light power of the SWM. This is the same as the well-known mode competition. Howevre, when the wavelength separation is increased to $111 \mathrm{~nm}$ (see Fig. 4 (b)), a curve with positive gradient appears on the plane. That is, increasing the light power of the SWM leads to the increase of the light power of the LWM. This behavior is opposite to mode competition, so we call it mode anti-competition. Some experimental results for larger wavelength separation are shown

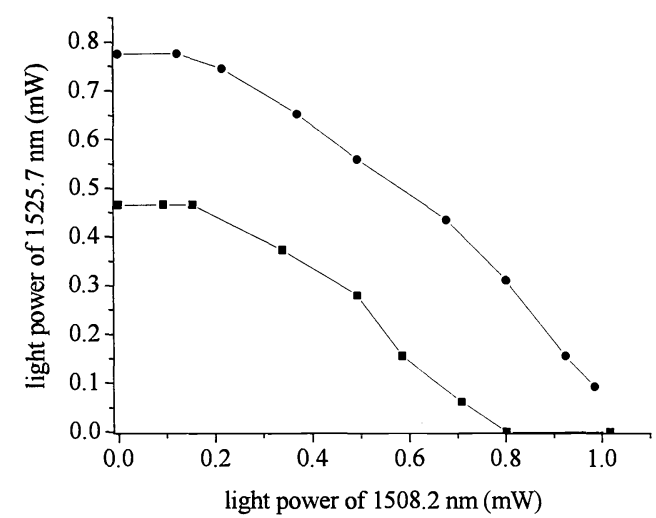

(a)

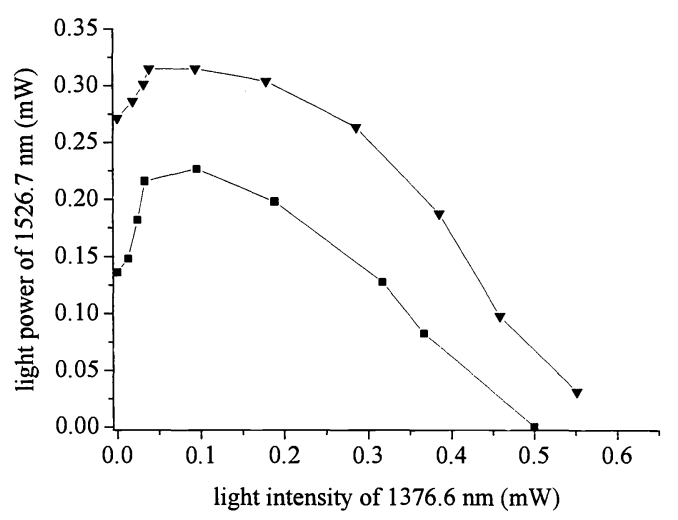

(c)

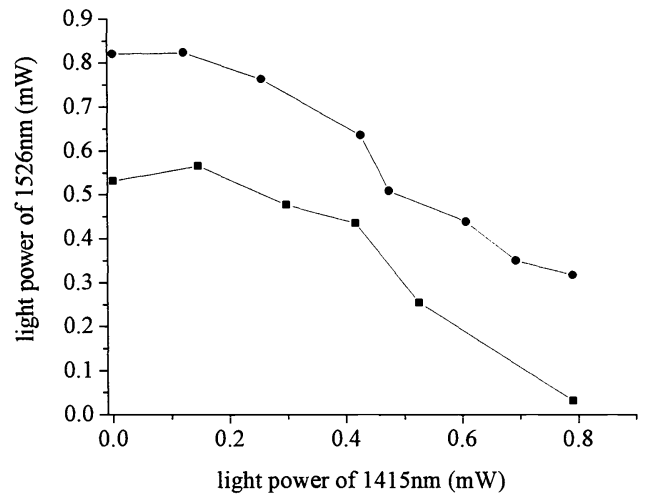

(b)

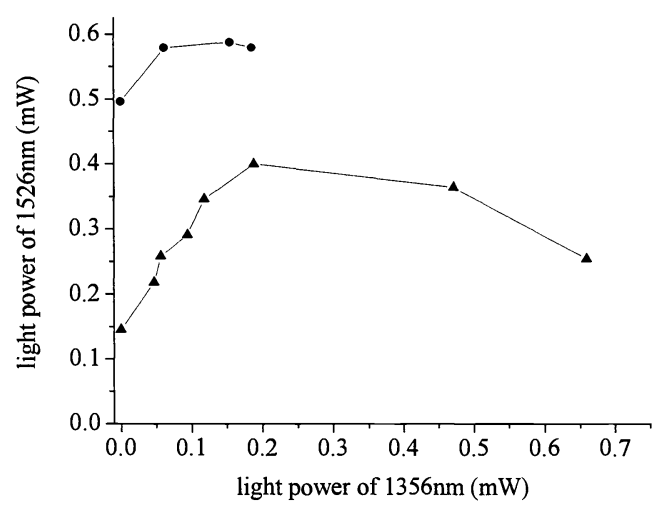

(d)

Fig. 4. Variation of the light power of the LWM to that of the SWM at wavelength separation of: (a) $18 \mathrm{~nm}$, (b) $111 \mathrm{~nm}$, (c) $150 \mathrm{~nm}$, (d) $168 \mathrm{~nm}$. 
in Fig. 4 (c), (d).

Next, some influential factors of anti-competition are investigated. The first one is wavelength separation of the two modes. From Fig. 4, we can see that at larger wavelength separation, the slope of the positive-gradient curve is steeper, and the maximum power the LWM can reach under fixed initial power is larger. That is, the larger the wavelength separation is, the severer anti-competition is. For example, when the initial power of the LWM (the power of the LWM when the power of the SWM is zero) is fixed to about $0.14 \mathrm{~mW}$, anti-competition can cause the light power of the LWM to increase to about $0.23 \mathrm{~mW}$ and $0.4 \mathrm{~mW}$ at wavelength separation $150 \mathrm{~nm}$ and $168 \mathrm{~nm}$, respectively (see Fig. 4 (c), (d)). In addition, for those two curves, the slopes of the positive gradient region are about 1.5 and 1.8 at wavelength separation of $150 \mathrm{~nm}$ and $168 \mathrm{~nm}$, respectively.

The second influential factor is the light power of the SWM. From Fig. 4, we see that no matter which wavelength separation is, anti-competition can exist only when the light power of the SWM is below certain level. For example, at wavelength separation of $150 \mathrm{~nm}$ and $168 \mathrm{~nm}$ (see Fig. 4 (c), (d)), anti-competition can exist only when the light power of the SWM is below $0.1 \mathrm{~mW}$ and $0.2 \mathrm{~mW}$, respectively. Above this level, anti-competition disappears, and the interaction between these two modes gradually turns into competition. In general, this power level, or say, the existing range of a anti-competition, is larger at large wavelength separation. During the transition from anti-competition to competition, region exists where the variation of the SWM power has no influence on the LWM power. The existing range of anti-competition seems larger at wavelength separation $111 \mathrm{~nm}$ than at $150 \mathrm{~nm}$. This is because, at wavelength separation $111 \mathrm{~nm}$, we only sample two points when the power of the short-wavelength mode increases from $0 \mathrm{~mW}$ to $0.18 \mathrm{~mW}$ (see Fig. 4 (b)). Therefore, in Fig. 4 (b), the maximum power of the long-wavelength mode (or say, the end point of anti-competition) should have appeared prior to our second sample point, but that point was missed.

The third influential factor is the initial power of the LWM. In general, anti-competition is severer when the initial power of the LWM is smaller. This can be verified from Fig. 4 (b), (c), (d).

The last influential factor of anti-competition is the wavelength position of the two modes. As shown in Fig. 5, anti-competition at fixed wavelength separation $160 \mathrm{~nm}$ can have different behaviors because of different wavelength positions. When both modes reside in the longer wavelength region, anti-competition is severer. The same phenomenon can be observed for wavelength separation larger than $140 \mathrm{~nm}$. When the wavelength separation is smaller than $140 \mathrm{~nm}$, a phenomenon behaves in the opposite way appears. That is, when both modes reside in the longer wavelength region, anti-competition is less obvious.

\subsection{Reasons for anti-competition}

The reasons for anti-competition are as follows. First, as discussed above, the emission spectrum of the laser gain medium is the overlap of the emission spectra of two different QWs. Since in our experiment, the wavelength position of the LWM is always above $1510 \mathrm{~nm}$ (see Fig. 4, 5), most of the carriers for the oscillation of the LWM are mostly

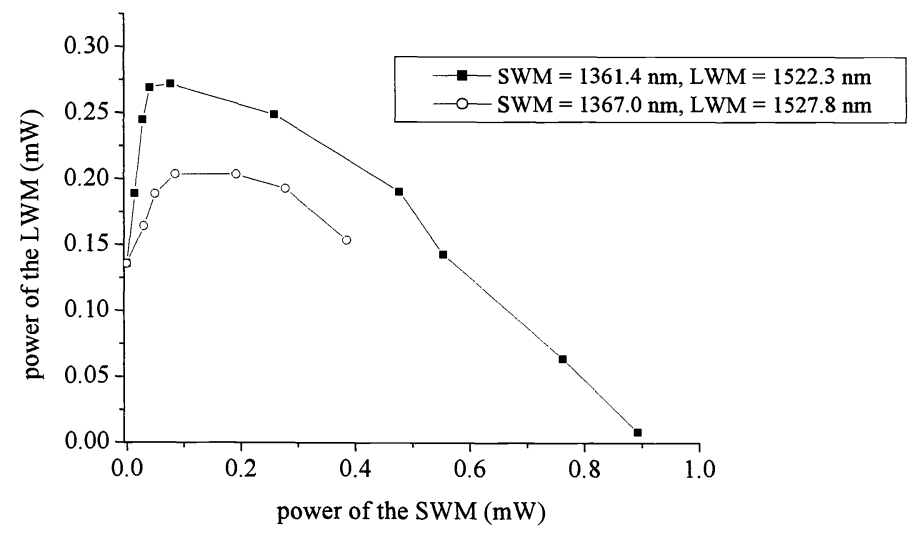

Fig. 5. Power of the LWM - power of the SWM curve with wavelength separation fixed to $160 \mathrm{~nm}$. 
contributed from the $\mathrm{n}=1$ state of the 8.7-nm $\operatorname{In}_{0.53} \mathrm{Ga}_{0.47}$ As QWs. However, the wavelength position of the SWM ranges from $1356 \mathrm{~nm}$ to $1415 \mathrm{~nm}$ at wavelength separation ranging from $168 \mathrm{~nm}$ to $111 \mathrm{~nm}$. Therefore, both the $6.0-\mathrm{nm}$ $\mathrm{In}_{0.67} \mathrm{Ga}_{0.33} \mathrm{As}_{0.72} \mathrm{P}_{0.28}$ QWs and the $\mathrm{n}=2$ state of the 8.7-nm $\mathrm{In}_{0.53} \mathrm{Ga}_{0.47} \mathrm{As}$ QWs contribute to the carriers for the oscillation of the SWM. The ratio of the carrier contribution form these two types of QWs to the SWM depends on the wavelength position of the SWM. If the SWM is located near $1300 \mathrm{~nm}$, the $\operatorname{In}_{0.67} \mathrm{Ga}_{0.33} \mathrm{As}_{0.72} \mathrm{P}_{0.28}$ QWs have more contribution to the gain, and vice versa. Therefore, at wavelength separation $111 \mathrm{~nm}$ (see Fig. 4 (b)), the two wavelengths are nearly contributed from the $\mathrm{In}_{0.53} \mathrm{Ga}_{0.47} \mathrm{As}$ QWs. On the other hand, at wavelength separation $168 \mathrm{~nm}$ (see Fig. 4 (d)), the SWM and LWM are nearly contributed from the $\operatorname{In}_{0.67} \mathrm{Ga}_{0.33} \mathrm{As}_{0.72} \mathrm{P}_{0.28}$ QWs and the $\operatorname{In}_{0.53} \mathrm{Ga}_{0.47} \mathrm{As}$ QWs, respectively. Therefore, competition at wavelength separation $168 \mathrm{~nm}$ is weaker than at $111 \mathrm{~nm}$ (as shown in Fig. 4) because the carrier transportation between different QWs is a relatively slow process, compared with the intraband relaxation in the same well.

The second reason is, because the photon energy of the short-wavelength QWs is larger than that of the longwavelength QWs, some of the emitted short-wavelength photons will be absorbed by the long-wavelength QWs. This can provide the long-wavelength QWs with some optical gain, and is somewhat similar to optical pumping. At larger wavelength separation, the photon energy difference is larger, so stronger optical pumping and thus severer anticompetition occurs. In addition, if the initial power of the LWM is large, there are already plenty carriers for the oscillation of LWM. Therefore, optical pumping and anti-competition is weak at large LWM initial power. Moreover, the gain spectrum of the $\mathrm{In}_{0.53} \mathrm{Ga}_{0.47} \mathrm{As} \mathrm{QW}$ has one peak at $1480 \mathrm{~nm}$ [24]. That is, the gain at $1522.3 \mathrm{~nm}$ is larger than at

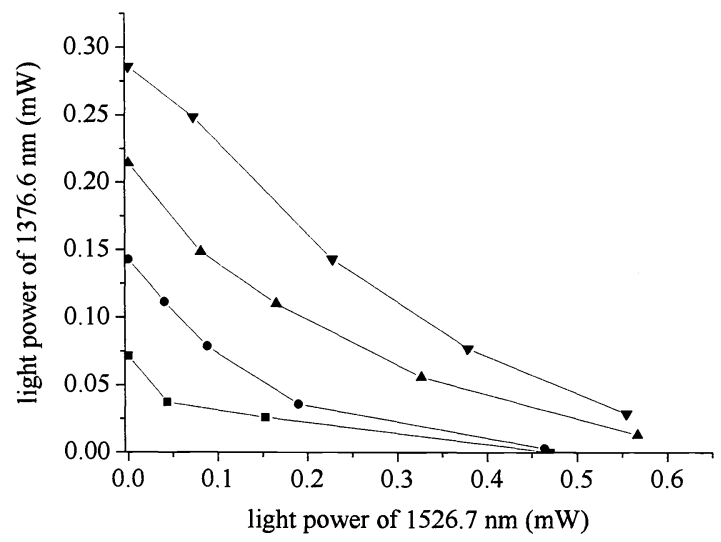

Fig. 6. Variation of the light power of the SWM to that of the LWM at wavelength separation of $150 \mathrm{~nm}$. The wavelength positions of the two modes are the same as in Fig. 4 (c).

$1527.8 \mathrm{~nm}$, and thus $1527.8 \mathrm{~nm}$ has fewer carriers. This results in that $1527.8 \mathrm{~nm}$ has stronger "optical consumption ability" of the short-wavelength photons. Therefore, LWM at $1527.8 \mathrm{~nm}$ will have less apparent anti-competition behavior than LWM at $1527.8 \mathrm{~nm}$ under fixed wavelength separation, as shown in Fig. 5.

Since anti-competition is due to the physics similar to optical pumping, it can exist only at the LWM. For the SWM, increasing the light power of the LWM will only decrease its light power, as shown in Fig. 6 for the same two modes as in Fig. 4 (c). Note that although both Fig. 4 (a) and Fig. 6 are curves with competition, their behaviors are different. For example, the curves in Fig. 4 (a) are curving downward, whereas those in Fig. 6 are curving upward.

\section{ANALYSIS}

Previous models of competition usually use the following rate equations to describe the gain saturation: [5]

$$
\frac{d I_{1}}{d t}=\left(\frac{g_{10}}{1+S_{1} I_{1}+C_{12} I_{2}}-l_{1}\right) I_{1},
$$




$$
\frac{d I_{2}}{d t}=\left(\frac{g_{20}}{1+S_{2} I_{2}+C_{12} I_{1}}-l_{2}\right) I_{2},
$$

where the first term in parentheses [Eq. (1a)] represents the gain with self- and cross-saturation; $g_{10}$ and $g_{20}$ are the unsaturated gains of the modes $I_{1}$ and $I_{2}$, respectively; $l_{1}$ and $l_{2}$ are losses of the modes $I_{1}$ and $I_{2}$, respectively. In reality, the denominators in Eqs. (1a) and (1b) are not linear functions of $I 1$ and $I 2$. The rate equations can be put into more general forms:

$$
\begin{aligned}
& \frac{d I_{1}}{d t}=\left[G_{1}\left(I_{1}, I_{2}\right)-l_{1}\right] I_{1}, \\
& \frac{d I_{2}}{d t}=\left[G_{2}\left(I_{1}, I_{2}\right)-l_{2}\right] I_{2},
\end{aligned}
$$

with $G_{1}\left(I_{1}, I_{2}\right)$ and $G_{2}\left(I_{1}, I_{2}\right)$ representing general relations of the gains and the intensities $I_{1}$ and $I_{2}$. In the steady state, $d I_{1} / d t=d I_{2} / d t=0$. Equations (2a) and (2b) can then be written as

$$
\begin{aligned}
& {\left[G_{1}\left(I_{1}, I_{2}\right)-l_{1}\right] I_{1}=0,} \\
& {\left[G_{2}\left(I_{1}, I_{2}\right)-l_{2}\right] I_{2}=0,}
\end{aligned}
$$

where $G_{1}\left(I_{1}, I_{2}\right)-l_{1}=0$ and $G_{2}\left(I_{1}, I_{2}\right)-l_{2}=0$ represent two curves on the $I_{1}-I_{2}$ phase plane.

Let's define $I_{1}$ and $I_{2}$ as the intensity of the LWM and SWM, respectively. The saturation effect causes the gain to decrease with the intensity, so the self-saturation leads to $\partial G_{2} / \partial I_{2}<0$. The competition is due to cross-saturation $[2,3]$, so $\partial G_{2} / \partial I_{1}<0$. Thus, for competition situation, the gain curve $G_{2}\left(I_{1}, I_{2}\right)-l_{2}=0$ should have the slope $d I_{1} /$ $d I_{2}<0$, which is the case for Fig. 4 (a) and for previous experiments [2,6]. For the case of anti-competition shown in Fig. 4 (b) (d), $d I_{1} / d I_{2}>0$. Because self-saturation still gives $\partial G_{2} / \partial I_{2}<0$, it must be that $\partial G_{2} / \partial I_{1}>0$. As a result, the power-series expansion of $G_{2}\left(I_{1}, I_{2}\right)$ consists of a term $\left(\partial G_{2} / \partial I_{1}\right) I_{1}\left(=\gamma I_{1}>0\right)$, which means the condition like optical pumping. That is, the short-wavelength mode gives away its optical power to the long-wavelength mode. However, the two-mode operation is not exactly the same as optical pumping. As shown in Fig. 4 (b) (d), some regime of competition still exists, indicating that the cross-saturation plays an important role again for certain intensity of $I 1$.

\section{CONCLUSION}

Anti-competition of laser modes is observed in semiconductor laser with nonidentical MQWs. In this behavior, the oscillation intensity of the long-wavelength mode can be enhanced by that of the short-wavelength mode, which is opposite to the well-known mode competition. The phenomenon of anti-competition can be observed as long as the wavelength separation is larger than $111 \mathrm{~nm}$, and is even more prominent for wavelength separation up to $168 \mathrm{~nm}$. The influential factors of anti-competition includes the wavelength separation, the power of both modes and the wavelength position. Theoretical analysis shows that anti-competition is due to the physics similar to optical pumping.

\section{REFERENCES}

[1] W. E. Lamb, "Theory of an optical maser," Phys. Rev. A 134, 1429 (1964)

[2] A. E. Siegman, Lasers (University Science Books, 1986).

[3] K. Shimoda, Introduction to Laser Physics (Springer, Berlin, 1984), p.187.

[4] M. Watanabe, H. Itoh, S. Mukai, and H. Yajima, "Optical tristability using a twin-stripe laser diode," Appl. Phys. Lett. 50, 427 (1987). 
[5] C.-F. Lin and P.-C. Ku, "Analysis of stability in two-mode laser system,"IEEE J. Quantum Electron. 32, 1377 (1996).

[6] H. Kawaguchi, Bistability and Nonlinearities in Laser Diodes (Artech House, Norwood, MA, 1994), p. 199.

[7] S. Sivaprakasam, P. S. Spencer, P. Rees, and K. A. Shore, "Regimes of chaotic synchronisation in external cavity laser diodes," IEEE J. Quantum Electron. 38, 1155 (2002).

[8] H. Kawaguchi and I. S. Hidayat, "Gigahertz all-optical flip-flop operation of polarisation-bistable vertical-cavity surface emitting lasers," Electron. Lett. 31, 1150 (1995).

[9] G. D. VanWiggeren and R. Roy, Science (Washington, DC, U.S.) 279, 1198 (1998).

[10] X. Jin and S. L. Chuang, "Relative intensity noise characteristics of injection-locked semiconductor lasers ," Appl. Phys. Lett. 77, 1250 (2000).

[11] H. Statz and C. L. Tang, J. Appl. Phys. 36, 3923 (1965).

[12] L. E. Hargrove, R. L. Fork, and M. A. Pollack, "Locking of He-Ne Laser Modes Induced by Synchronous Intracavity Modulation," Appl. Phys. Lett. 5, 4 (1964).

[13] P. W. Smith, Proc. IEEE 58, 1342 (1970).

[14] I. White, R. Penty, M. Webster, Y. J. Chai, A. Wonfor, and Sadegh Shahkooh, "Wavelength switching components for future photonic networks," IEEE Communications Magazine 40, 74 (2002).

[15]H. S. Gingrich, D. R. Chumney, S.-Z. Sun, S. D. Hersee, L. F. Lester, and S. R. Brueck, "Broadly-tunable external cavity laser diodes with staggered thickness multiple quantum wells," IEEE Photon. Technol. Lett. 9, 155 (1997).

[16]C.-F. Lin and B.-L. Lee, "Extremely broadband AlGaAs/GaAs superluminescent diodes," Appl. Phys. Lett. 71,1598 (1997).

[17]N. Tessler and G. Eisenstein, "On carrier injection and gain dynamics in quantum well lasers," IEEE J. Quantum Electron. 29, 1586 (1993).

[18]R. Nagarajan, T. Fukushima, S. W. Corzine, and J. E. Bowers, "Effects of carrier transport on high-speed quantum well lasers," Appl. Phys. Lett. 59, 1835 (1991).

[19]H. Yamazaki, A. Tomita, M. Yamaguchi, and Y. Sasaki, "Evidence of nonuniform carrier distribution in multiple quantum well lasers," Appl. Phys. Lett. 71, 767 (1997).

[20]B.-L. Lee, C.-F. Lin, J.-W. Lai, and W. Lin, "Experimental evidence of nonuniform carrier distribution in multiplequantum well laser diodes," Electron. Lett. 34, 1230 (1998).

[21]D. Ban and E. H. Sargent, "Influence of nonuniform carrier distribution on the polarization dependence of modal gain in multiquantum-well lasers and semiconductor optical amplifiers," IEEE J. Quantum Electron. 36, 1081 (2000).

[22]C.-F. Lin, B.-R. Wu, L.-W. Laih, and T.-T. Shih, "Sequence influence of nonidentical InGaAsP quantum wells on broadband characteristics of semiconductor optical amplifiers-superluminescent diodes," Optics Lett. 26, 1099 (2001).

[23]D.Ahn and S. L. Chuang, "Optical gain and gain suppression of quantum-well lasers with valence band mixing," IEEE J. Quantum Electron. 26, 13 (1990).

[24]C.-S. Chang and S. L. Chuang, "Modeling of strained quantum-well lasers with spin-orbit coupling," IEEE Journal of Selected topics in Quantum Electronics 1, 218 (1995). 